\title{
The USP8 mutational status may predict drug susceptibility in corticotroph adenomas of Cushing's disease
}

\author{
Kyohei Hayashi ${ }^{1,2}$, Naoko Inoshita ${ }^{2}$, Kohei Kawaguchi ${ }^{3}$, Arif Ibrahim Ardisasmita ${ }^{3}$, \\ Hisanori Suzuki ${ }^{1}$, Noriaki Fukuhara ${ }^{4}$, Mitsuo Okada ${ }^{4}$, Hiroshi Nishioka ${ }^{4,5}$, \\ Yasuhiro Takeuchi, ${ }^{1,5}$, Masayuki Komada ${ }^{3}$, Akira Takeshita ${ }^{1,5}$ and Shozo Yamada ${ }^{4,5}$ \\ Departments of ${ }^{1}$ Endocrinology and Metabolism and ${ }^{2}$ Pathology, Toranomon Hospital, 2-2-2 Toranomon, Minato, \\ Tokyo 105-8470, Japan, ${ }^{3}$ Department of Biological Sciences, Tokyo Institute of Technology, $4259-\mathrm{B} 16$ Nagatsuta, \\ Midori, Yokohama 226-8501, Japan, ${ }^{4}$ Department of Hypothalamic and Pituitary Surgery, Toranomon Hospital, \\ 2-2-2 Toranomon, Minato, Tokyo 105-8470, Japan and ${ }^{5}$ Okinaka Memorial Institute for Medical Research, \\ 2-2-2 Toranomon, Minato, Tokyo 105-8470, Japan
}

Correspondence should be addressed to A Takeshita Email coactivator@mac.com

\begin{abstract}
Context: Somatic mutations in the ubiquitin-specific peptidase USP8 gene were recently detected in one- to two-third(s) of corticotroph adenomas of Cushing's disease (CD). These mutations may lead to the deubiquitination of EGFR, thereby increasing EGFR signaling, which has been implicated in ACTH hypersecretion.

Objective: Our objective was to determine the impact of USP8 mutations on the clinicopathological features of CD. Subjects and methods: USP8 mutations as well as clinicopathological characteristics were examined in 60 corticotroph adenomas including 15 Crooke's cell adenomas (CCAs), a rare histological variant presenting with generally aggressive behavior, using qRT-PCR and/or immunohistochemistry.

Results: USP8 mutations were exclusively detected in women, except for one case, with a prevalence of $42.2 \%$ in non-CCA and $13.3 \%$ in CCA (overall $35 \%$ ). Clinically well-behaved presentations including microadenoma and curative resection were more common in mutated cases. The expression of EGFR was not associated with the mutation status. In contrast, mutated tumors expressed significantly higher levels of POMC, SSTR5, and MGMT.

Conclusions: Microadenomas that strongly express POMC were common among mutated tumors, which may lead to the mechanisms by which very small adenomas secrete excess ACTH to present overt CD. While USP8 mutations were less likely to enhance tumorous ACTH hypersecretion via EGFR-mediated activation, the presence of USP8 mutations may predict favorable responses to the somatostatin analog pasireotide, which exhibits high affinity for SSTR5. In contrast, non-mutated aggressive tumors such as CCA may respond better to the alkylating agent temozolomide because of their significantly weak expression of MGMT.
\end{abstract}

\section{Introduction}

Cushing's disease (CD) is caused by an ACTH-secreting corticotroph adenoma that induces hypercortisolism. Most ACTH-secreting adenomas are microadenomas ( $<10 \mathrm{~mm}$ in maximum diameter), while macroadenomas $(\geq 10 \mathrm{~mm}$ ) are detected in $10-20 \%$ of patients. The firstline treatment for $\mathrm{CD}$ is transsphenoidal surgery (TSS).
The selective surgical removal of a microadenoma generally results in a cure with a low recurrence rate. In contrast, macroadenomas are typically more invasive tumors and often recur after surgery.

Crooke's cell adenoma (CCA), a histological variant type of corticotroph adenomas, is characterized by a

Published by Bioscientifica Ltd. 
prominent hyalinized cytoplasm (Crooke's hyaline change) in most adenoma cells. Although CCA is a rare subtype of CD with an estimated prevalence of $4.4-14 \%$ among all corticotroph adenomas, it is generally aggressive, presenting as invasive macroadenomas, is resistant to surgery and radiotherapy, and has a high recurrence rate. The development of corticotroph carcinomas in preexisting CCA is not uncommon $(1,2)$.

The four members of the ErbB family, which includes epidermal growth factor receptor (EGFR) and ERBB2-4, are cell surface receptor tyrosine kinases (TKs) (3). When tumor cells overexpress ErbB receptors, they exhibit aggressive tumor cell growth. Previous studies reported that pituitary adenomas including corticotroph adenomas had variable EGFR expression $(4,5)$, which correlated with tumor aggressiveness $(6,7,8)$. Somatic mutations in the 14-3-3 protein binding motif of the deubiquitinating enzyme (DUB) gene, ubiquitin specific peptidase 8 (USP8), were recently detected in approximately one- to two-third(s) of corticotroph adenomas (9, 10). These mutations potentially lead to the deubiquitination of EGFR, thereby increasing EGFR signaling, which has been implicated in corticotroph tumorigenesis. Furthermore, in vitro studies revealed that USP8 mutations enhanced the promoter activity of the gene encoding proopiomelanocortin (POMC) through stabilized EGFR signaling, which may explain ACTH hypersecretion in patients with $\operatorname{CD}(9,10,11)$. These findings have raised the question as to whether USP8 mutations are associated with the clinical features of $\mathrm{CD}$ such as tumor aggressiveness, ACTH hypersecretion, surgical remission rate, and drug susceptibility to the dopamine agonist cabergoline and somatostatin analog pasireotide $(12,13)$, as well as the alkylating agent temozolomide, which is used in the treatment of aggressive pituitary adenomas and carcinomas $(2,14,15,16)$.

Clinical presentation such as the tumor size, extent of cavernous sinus (CS) invasion, surgical outcome, and Ki-67 labeling index has been associated with tumor aggressiveness $(15,16)$. The positive expression of dopamine receptor D2 (DRD2), somatostatin receptors (SSTRs), and EGFR have been shown to predict drug susceptibility to dopamine agonists, somatostatin analogs, and TK inhibitors respectively $(12,13,17)$. In contrast, the negative expression of $\mathrm{O}^{6}$-methylguanine DNA methyltransferase (MGMT) and the DNA mismatch repair protein MSH6 have been associated with tumor responsiveness $(18,19)$ and resistance to temozolomide (20) respectively. In order to determine the impact of USP8 mutations in CD, we herein evaluated the clinicopathological features of 60 corticotroph adenomas including 15 CCAs using quantitative real-time PCR (qRT-PCR) and/or immunohistochemistry, and evaluated their relationships with the USP8 mutation status.

\section{Subjects and methods}

\section{Patients}

We reviewed all tumor samples from 209 patients with clinically active CD who underwent TSS between March 2004 and October 2013. In all cases, surgery was performed by the same surgeon (SY) who has experience with more than 2500 pituitary surgeries. Samples were examined by routine histological methods and immunohistochemical staining as previously described $(21,22)$. They were classified as CCA when the percentage of Crooke's hyaline change in tumor cells was $>50 \%$, with the remaining tumor samples being considered as nonCrooke's cell adenoma (non-CCA) $(1,21)$. Sixteen tumor samples were identified as CCA from 209 samples $(7.7 \%)$, and 15 out of 16 samples were available for RNA extraction. They were compared with 45 tumor samples selected from recent non-CCA tumor samples. The control group comprised three normal anterior pituitary tissues obtained from patients during surgery for craniopharyngioma without hypopituitarism preoperatively. Informed consent was obtained from all patients and tissue collection was approved by the Institutional Review Board of Toranomon Hospital. Preoperative contrastenhanced MRI (1.5 or 3.0 T) was performed in all patients. CS invasion was confirmed by both operative and histological findings. Patients were considered to be in surgical remission on the basis of postoperative serum cortisol levels $<3 \mu \mathrm{g} / \mathrm{dl}$ (23).

\section{RNA extraction and CDNA preparation}

Pituitary tumor samples were stored in RNAlater (Life Technologies) at $-80^{\circ} \mathrm{C}$ until RNA extraction. Total RNA was prepared using RNeasy mini (Qiagen). PrimeScript RT reagent (Takara Bio, Otsu, Japan) was used for the preparation of cDNA.

\section{qRT-PCR}

With the resulting cDNA as a template, qRT-PCR was performed with Power SYBR Green PCR Master Mix (Life Technologies) and gene-specific primers (see Supplementary Table 1 , see section on supplementary data given at the end of this article) in a StepOnePlus real-time PCR 
system (Life Technologies). Relative quantification was performed using standard curve methods. mRNA expression results were normalized to GAPDH and expressed in arbitrary units (AU).

\section{Direct sequencing for detection of USP8 mutations}

Tumor cDNA was amplified by PCR using the forward primer, 5'-GGGAGGATCGTACCAGGACT-3' in exon 13 and the reverse primer 5'-AGGTTCCGAATCTGAGAAGCA-3'in exon 14, corresponding to codon 645-764 of USP8 (GenBank accession number: NM_005154.4). Direct sequencing was performed using internal primers.

\section{Biochemistry}

All experimental procedures regarding in vitro experiments on COS-7 and HeLa cells, including the construction of cDNA expression plasmids, cell culture, DNA transfection, immunoprecipitation and immunoblotting, DUB assays, and immunofluorescence staining were performed as previously described (9).

\section{Immunohistochemistry}

A summary of the primary antibodies used and working dilutions are provided in Supplementary Table 2, see section on supplementary data given at the end of this article. Slides were stained using an EnVision+SystemHRP Kit (Dako, Glostrup, Denmark). Immunohistochemical reactions were developed with diaminobenzidine and slides were counterstained with hematoxylin.

EGFR, SSTR2, SSTR5, DRD2, MGMT, and MSH6 protein expression levels were scored using the immunoreactive score (IRS) as previously reported $(24,25)$; IRS (0-12) was the product of the proportion of immunoreactive cells $(0,0 \% ; 1,<10 \% ; 2,10-50 \% ; 3,51-80 \%$; or 4 , $>80 \%)$ multiplied by the staining intensity $(0$, no staining; 1 , weak; 2 , moderate; and 3 , strong). The scores of the images are shown in Fig. 1.

\section{Statistical analysis}

Data were expressed as a median with an interquartile range (IQR). Comparisons between categorical variables were performed using Fisher's exact test. Comparisons between numerical variables were performed using the Mann-Whitney $U$ test. Correlations were determined by calculating Spearman's rank correlation coefficient. $P$ values of $<0.05$ were considered significant. A stepwise forward-selection multivariate regression analysis was employed with either surgical remission, mRNA expression, or the IRS score as a dependent variable and different parameters including the USP8 mutation status, USP8 mRNA expression, pathological finding (non-CCA or CCA), sex, plasma ACTH concentration, maximum tumor diameter, Knosp classification, cavernous sinus invasion, and Ki-67 labeling index as independent variables $(n=51$, after excluding nine subjects whose tumorous RNA samples may have been contaminated with a significant amount of the normal anterior pituitary).

\section{Results}

\section{Clinical characteristics of subjects}

A previous in vitro study suggested that USP8 mutations were associated with EGFR activation of corticotroph adenomas. Therefore, we speculated that the aggressive type of CD, CCA may be more likely to demonstrate USP8 mutations such that EGFR protein expression levels may be higher in CCA than in non-CCA. Therefore, we evaluated 60 samples comprising 15 CCA and 45 nonCCA. The ratio of CCA (25\%) in the present study was markedly higher than the actual prevalence of CCA (7.7\%) in our hospital.

The clinical characteristics of the tumors examined are summarized in Table 1 . Note that specimens of very small microadenomas, such as $<5 \mathrm{~mm}$, were generally used for diagnostic purposes only, and were rarely available for the present study, lending a certain selection bias to the nonCCA group. CCA were significantly larger than non-CCA (maximum tumor diameter, median $19.2 \mathrm{~mm}$ vs $9.4 \mathrm{~mm}$, $P=0.002$ ) (Supplementary Figure 1A, see section on supplementary data given at the end of this article). Macroadenomas were more frequent among CCA than among non-CCA (93.3\% vs 40\%, $P<0.001)$. The prevalence of CS invasion was significantly higher in CCA than in non-CCA ( $80 \%$ vs $48.9 \%, P=0.041)$. The surgical remission rate was lower in CCA than in non-CCA although there was no statistic difference $(46.7 \%$ vs $75.6 \%, P=0.055)$. Clinical features including age at diagnosis, sex ratio, plasma ACTH, serum cortisol, the Ki-67 labeling index, and prior history of TSS were not significantly different between the two groups. Taken together, our CCA cases confirmed the aggressive clinical features of invasive macroadenomas with a low surgical remission rate. 




\section{Figure 1}

Examples of immunoreactive scores (IRS, 0-12) for EGFR, SSTR2, SSTR5, DRD2, MGMT, and MSH6. Sections were counterstained with hematoxylin. The highest IHC scores for EGFR and DRD2 were $6+$ and $8+$ respectively. Positive controls (colon cancer for EGFR and prolactinoma for DRD2) were included (original magnification, $\times 400$ ). 
Table 1 Comparisons of clinical characteristics of non-CCA vs CCA and WT vs USP8-mutated adenoma.

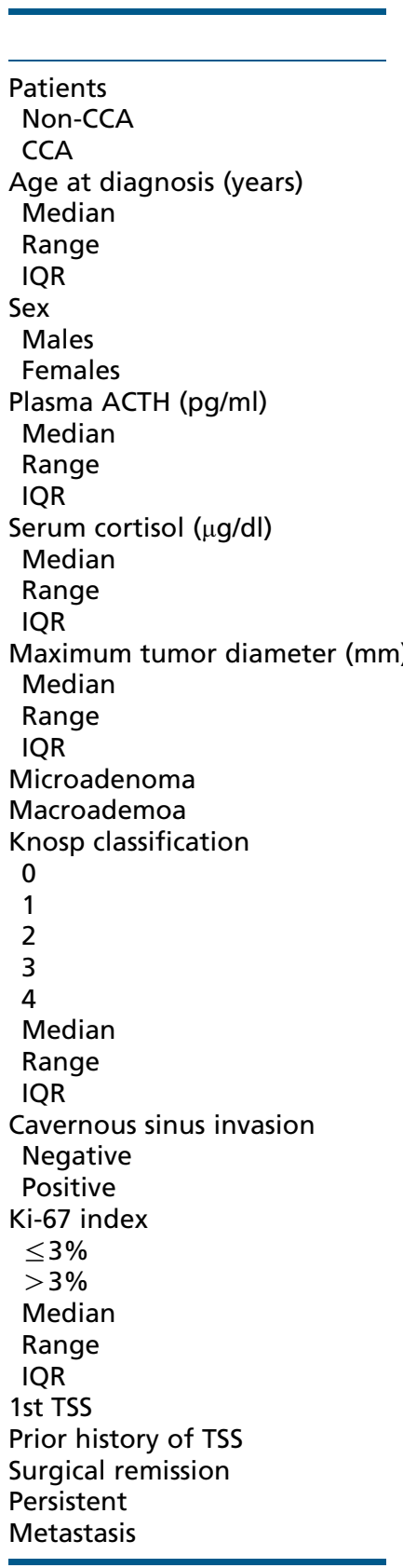

$\frac{\text { Total }}{60}$

$\frac{\text { Non-CCA }}{45(75 \%)} \frac{\text { CCA }}{15(25 \%)}$

$\boldsymbol{P}$

\begin{tabular}{c}
\hline $\mathbf{W T}$ \\
\hline 39 \\
$26(66.7 \%)$ \\
$13(33.3 \%)$
\end{tabular}

$\frac{\text { USP8 mutant }}{21}-$
$19(90.5 \%)$
$2(9.5 \%)$

$\frac{\boldsymbol{P}}{0.061^{\mathrm{a}}}$

$\begin{array}{ccc}45 & 41 & 45 \\ 19-81 & 19-65 & 22-81 \\ 36.3-57 & 28.5-51.5 & 37-59 \\ & & \\ 13(21.7 \%) & 9(20 \%) & 4(26.7 \%) \\ 47(78.3 \%) & 36(80 \%) & 11(73.3 \%)\end{array}$

$0.22^{\mathrm{b}}$

$$
\begin{gathered}
44 \\
22-81 \\
34-55
\end{gathered}
$$

$0.72^{a}$

$12(30.8 \%)$

$27(69.2 \%)$

102.75

27.3-1950

97.6

$70.1-141.8$

27.3-287.

17.4

9.7-54.0

$15.6-23.8$

19.7

$9.7-54.0$

15.7-27.6

10.7

3.5-45.6

$7.4-21.0$

$28(46.7 \%)$

$32(53.3 \%)$

9.4

3.5-45.6

$6.9-17.2$

$27(60 \%)$

$18(40 \%)$

$24(40 \%)$

$12(20 \%)$

$21(46.7 \%)$

$10(16.7 \%)$

$1(1.7 \%)$

$13(21.7 \%)$

1

0-4

0-2

$8(17.8 \%)$

$8(17.8 \%)$

$1(2.2 \%)$

$7(15.6 \%)$

1

0-4

0-2

$26(43.3 \%)$

$34(56.7 \%)$

$23(51.1 \%)$

$37(61.7 \%)$

$23(38.3 \%)$

2.7

$0.1-30.0$

$1.0-5.0$

$22(48.9 \%)$

$38(63.3 \%)$

$22(36.7 \%)$

$41(68.3 \%)$

$19(31.7 \%)$

$26(57.8 \%)$

$19(42.2 \%)$

2.9

$0.1-30.0$

1.0-5.0

$29(64.4 \%)$

$16(35.6 \%)$

$34(75.6 \%)$

$11(24.4 \%)$

0
111

78.1-228.0

$$
21.4
$$

12.5-44.6

17-30.2

19.2

4.9-40.3

14.1-25.5

$1(6.7 \%)$

$14(93.3 \%)$

$3(20 \%)$

$4(26.7 \%)$

$2(13.3 \%)$

$0(0 \%)$

$6(40 \%)$

2

0-4

$3(20 \%)$

$12(80 \%)$

110.1

27.3-1950

78.1-221.0

$0.52^{b}$

20.9

9.7-52.4

16.3-29.4

$0.0023^{b}$

16.3

4.0-45.6

8.4-25.5

$14(35.9 \%)$

$25(64.1 \%)$

$0.0003^{a}$

$25(64.1 \%)$

$0.053^{b}$

$12(30.8 \%)$
$8(20.5 \%)$
$6(15.4 \%)$
$0(0 \%)$
$13(33.3 \%)$
1
$0-4$
$0-4$

$12(57.1 \%)$

$4(19.0 \%)$

$4(19.0 \%)$

$1(4.8 \%)$

$0(0 \%)$

0

0-3

0-1

$0.041^{a}$

$14(35.9 \%)$

$25(64.1 \%)$

$12(57.1 \%)$

$9(42.9 \%)$

$24(61.5 \%)$

$13(61.9 \%)$

$8(38.1 \%)$

2.5

0.1-12.0

1.0-5.0

$0.5-20.0$

1.0-5.0

$6(40 \%)$

$7(46.7 \%)$

$8(53.3 \%)$

1
$9(60 \%)$

${ }^{\text {a}}$ Fisher's exact test.

${ }^{\mathrm{b}}$ Mann-Whitney U-test.

\section{Determination and relative mRNA expression of USP8 mutants}

The PCR-amplified cDNA fragments for codon 645-764 of USP8 containing the 14-3-3 binding motif were sequenced. USP8 mutations were detected in 21 (35\%) out of 60 adenomas. Sanger sequencing showed WT and mutant alleles in the mutated adenomas, consistent with heterozygous mutations. These mutations were S718del $(n=9)$, P720R $(n=6)$, S719P $(n=2)$, S718F $(n=1)$, S718_P720delinsT $(n=1)$, P720Q $(n=1)$, and P720_ D721del $(n=1)$ (Fig. 2A). Representative sequences of the mutations are shown in Supplementary Figure 2, see 
A

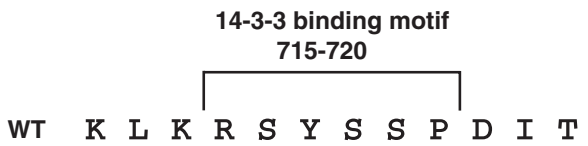

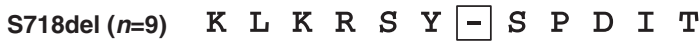
$\operatorname{P720R}(n=6) \quad K \quad L \quad K \quad R \quad S \quad Y \quad S \quad S \quad R \quad D \quad I \quad T$



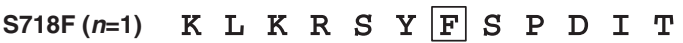

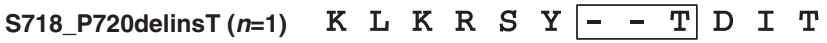
P720Q (n=1) K L K R $\quad S \quad Y \quad S \quad S \quad Q \quad D \quad I \quad T$

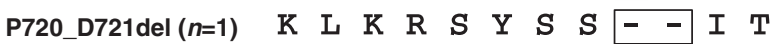

C USP8-Flag

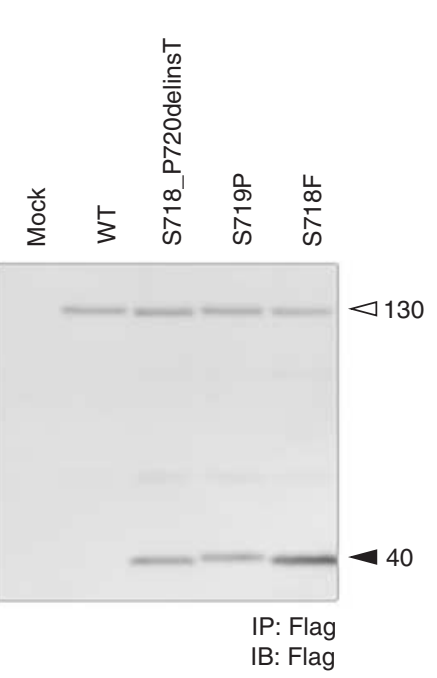

D

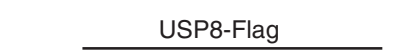

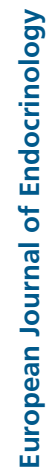

B

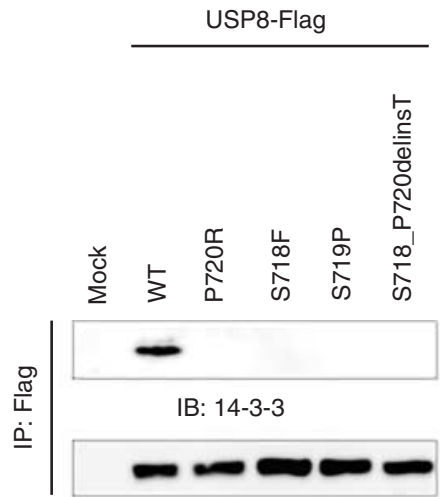

IB: Flag

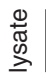

IB: 14-3-3

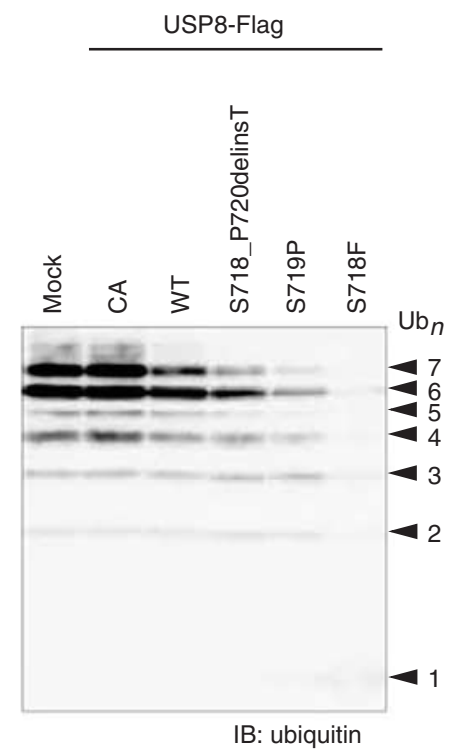

S718F

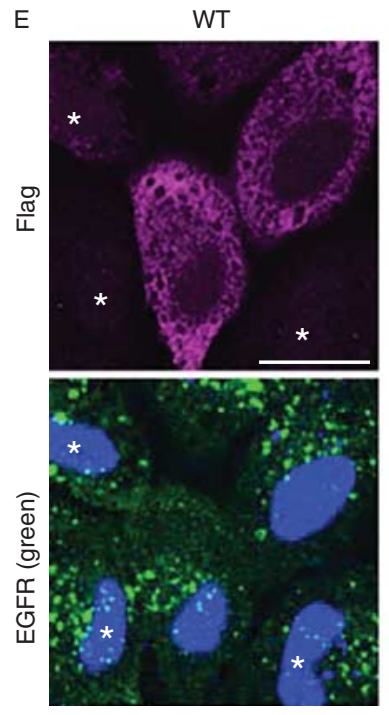

S718 P720delinsT
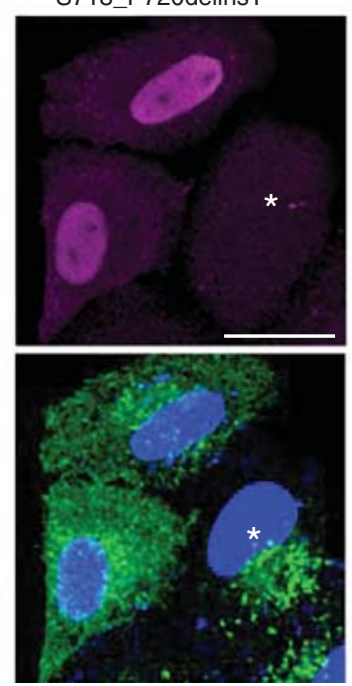

S719P

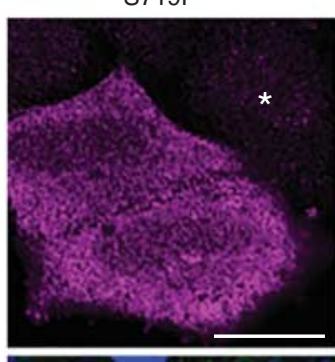

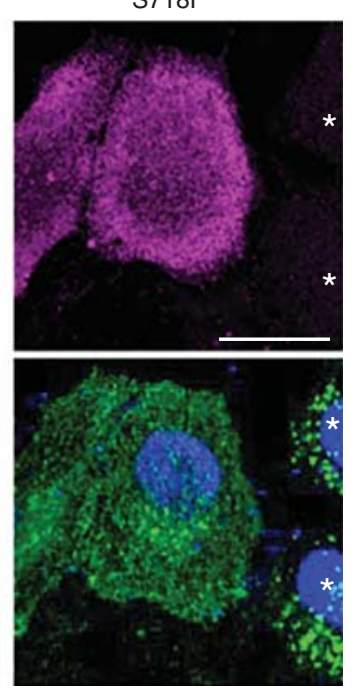


section on supplementary data given at the end of this article. S718_P720delinsT was a novel mutation. Others (S718del, P720R, S719P, S718F, P720Q, and P720_D721del) have been previously identified $(9,10,11)$.

USP8 mRNA levels were measured using qRT-PCR to determine whether the expression levels of USP8 differed between the USP8 mutants and the WT. In order to exclude the possibility of normal pituitary contamination in the RNA samples of 60 extracted corticotroph adenoma tissues, qRT-PCR was performed using primers specific for POUF1 (PIT1), which is expressed in thyro-mammosomatotroph-derived cells, but not in corticotroph lineages. Fifty-one samples (21 out of 26 WT in non-CCA, 15 out of 19 USP8 mutants in non-CCA, 13 out of 13 WT in CCA, and two out of two USP8 mutants in CCA) expressed $<2 \%$ POUF1 mRNA relative to the mean expression of three normal anterior pituitary samples, and were used in subsequent qRT-PCR analyses. However, the remaining nine RNA samples were excluded from subsequent qRTPCR analyses because they expressed more than 8\% POUF1 mRNA relative to the mean normal pituitary samples (Supplementary Figure 1B, see section on supplementary data given at the end of this article). Eight out of the nine excluded samples were microadenomas. USP8 mRNA expression was significantly higher in the mutants than in the WT USP8 (median 4.99 (AU) vs 2.59 (AU), $P=0.021$ ) (Supplementary Figure 1C).

\section{Functional analysis of USP8 mutations}

Previously-reported USP8 mutants loose binding to 14-3-3 proteins, undergo cleavage adjacent to the 14-3-3 binding motif to the $90-$ and $40-\mathrm{kDa}$ fragments, and the $40-\mathrm{kDa}$ fragment (C40) acquires high DUB activity $(9,11)$. Since the functions of three of the seven USP8 mutants identified in this study (S718F, S719P, and S718_P720delinsT) had not been determined, we performed a functional analysis. Co-immunoprecipitation experiments showed that 14-3-3 binding of C-terminally Flag-tagged S718F, S719P, and S718_P720delinsT was markedly affected, similar to the previously-characterized mutant P720R in mammalian COS-7 cells (Fig. 2B).

When the C-terminally Flag-tagged mutants were immunoprecipitated from transfected COS-7 cells and eluted from the antibody with the Flag peptide, cleaved C40 was detected in addition to the full-length $130-\mathrm{kDa}$ form in the three mutants but hardly in WT USP8 (Fig. 2C). C40 levels were consistently higher in S718F than in the other two mutants (Fig. 2D, left).

In order to determine DUB activity, Flag-tagged USP8 proteins were immunopurified from transfected COS-7 cells, as in Fig. 2C, and their purity, concentration, and partial cleavage were assessed by Coomassie staining (Fig. 2D, left). Approximately the same amounts of enzymes were incubated with Lys63-linked ubiquitin chains. Ubiquitin chains were more efficiently hydrolyzed by all USP8 mutants than by WT USP8 (Fig. 2D, right). Consistent with S718F being more efficiently cleaved than other mutants (Fig. 2D, left), it exhibited the highest DUB activity (Fig. 2D, right).

The effects of USP8 mutations on the downregulation of EGFR were examined in HeLa cells treated with $100 \mathrm{ng} / \mathrm{ml}$ EGF for $1 \mathrm{~h}$. While the overexpression of WT USP8 did not affect the endocytosis of ligand-activated

\section{Figure 2}

(A) Summarized diagram of somatic USP8 mutations in 21 out of 60 corticotroph adenomas. All mutations were detected between amino acids 718 and 721 in the hotspot region overlapping the 14-3-3 binding motif. (B) 14-3-3 protein binding of USP8 mutants. Top and middle) Anti-14-3-3 protein (top) and anti-Flag (middle) immunoblots of the anti-Flag immunoprecipitates of lysates of COS-7 cells expressing Flagtagged USP8 proteins. (Bottom) Anti-14-3-3 immunoblots of the lysates of COS-7 cells expressing Flag-tagged USP8 proteins. (C) Susceptibility of USP8 mutants to cleavage. Anti-Flag immunoblots of Flag-tagged USP8 proteins expressed in COS-7 cells, immunoprecipitated from their lysates with an anti-Flag antibody, and eluted from the antibody with the Flag peptide. Open and closed arrowheads indicate the full-length and

cleaved USP8 proteins respectively. (D) DUB activity of USP8 mutants. (Left) Coomassie staining of Flag-tagged USP8 proteins expressed in COS-7 cells and immunopurified from their lysates, as in (C). (Right) Anti-ubiquitin immunoblots of K63-linked ubiquitin chains (dimer-heptamer, Ub2-7) incubated with the immunopurified Flag-tagged USP8 proteins shown on the left. Arrowheads indicate each ubiquitin chain. (E) Effects of USP8 mutants on EGFR downregulation. Immunofluorescence staining of HeLa cells transfected with the Flag-tagged USP8 proteins and treated with $100 \mathrm{ng} / \mathrm{ml}$ EGF for $1 \mathrm{~h}$. Cells were triple-stained with an anti-Flag antibody (magenta, top), anti-EGFR antibody (green, bottom), and DAPI (blue, bottom). Asterisks indicate cells not expressing Flag-USP8 proteins. Scale bars, $10 \mu \mathrm{m}$. 
EGFR, that of the three new mutants led to the retention of EGFR on the plasma membrane, suggesting that elevated DUB activity in the mutants inhibited the downregulation of EGFR (Fig. 2E).

\section{Clinical characteristics of the USP8 mutant group}

The clinical features of the two groups, the WT and mutants, are summarized on the right side of Table 1 , while those of four groups, the WT and mutants in non-CCA or CCA, are summarized in Supplementary Table 3 , see section on supplementary data given at the end of this article. Mutations were more frequent in nonCCA (19 out of $45,42.2 \%$ ) than in CCA (two out of 15 , $13.3 \%$ ), and observed exclusively in females (20 out of 47 ), and rarely in males (one out of 13). Plasma ACTH levels were significantly lower in the mutant than in the WT group, whereas no significant difference was observed for serum cortisol. Tumors with USP8 mutations were significantly smaller than WT tumors, and all mutants were $<2 \mathrm{~cm}$ in size (Supplementary Figure 1A). Surgical remission was achieved in 20 out of 21 patients (95.2\%) in the mutant group, but in only 21 out of $39(53.8 \%)$ in the WT group (16 out of 26 (61.5\%) WT in non-CCA and five out of 13 (38.5\%) WT in CCA) (Supplementary Table 3). The stepwise forward-selection multivariate regression identified the maximum tumor diameter and USP8 mutation status as contributors to surgical remission (adjusted $R^{2}=0.493, P<0.0001$ ). No significant differences were observed in clinical features including age at diagnosis, the prevalence of CS invasion, and the Ki-67 labeling index between the WT and mutant groups. Taken together, USP8 mutations were typically detected in women and characterized as small non-aggressive tumors with a high surgical remission rate.

\section{Relative mRNA and protein expression of EGFR}

We determined the mRNA and protein expression levels of EGFR using qRT-PCR and Immunohistochemistry (IHC) respectively. No significant differences were observed in EGFR mRNA or IHC expression between WT and mutants (Fig. 3A and B), indicating that USP8 mutations did not affect the total protein level of EGFR. However, EGFR protein expression was significantly higher in CCA than in non-CCA (median IRS $1+$ vs $0, P=0.023$ ) (Fig. 3B), suggesting that the generally aggressive behavior of CCA was partly attributed to dominant EGFR protein expression. The stepwise regression analysis identified pathological findings (non-CCA or CCA) as the only
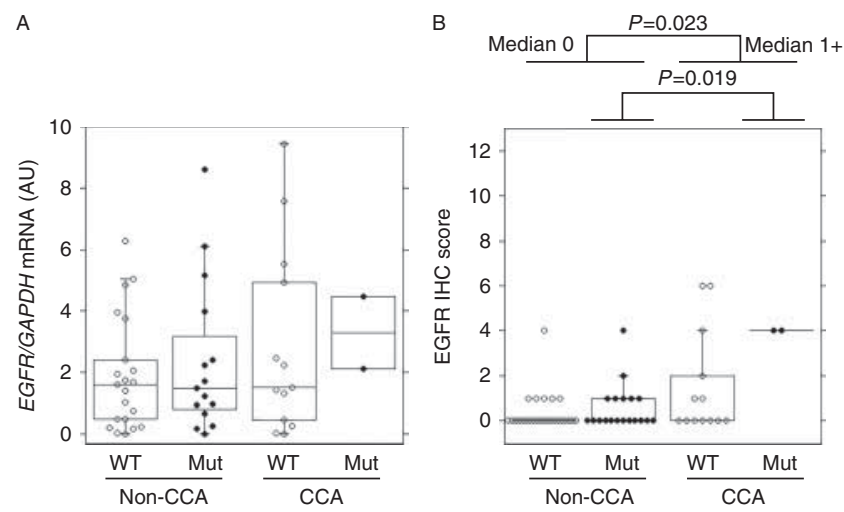

\section{Figure 3}

Expression of EGFR in corticotroph adenomas. (A) Comparison of EGFR mRNA expression levels between WT and USP8 mutant corticotroph adenomas in non-CCA or CCA. Expression levels of EGFR were analyzed by qRT-PCR, normalized to GAPDH, and expressed in arbitrary units (AU). (B) Comparison of EGFR IHC scores between WT and USP8 mutant corticotroph adenomas in non-CCA or CCA. EGFR staining intensity was scored using immunoreactive scores (IRS, $0-12$ ). Results are presented as box plots, and the line denotes the median values. The whiskers are 5th and 95th percentiles. Significance was determined by the Mann-Whitney $U$ test.

contributor to the IHC score of EGFR (adjusted $R^{2}=0.131, P=0.0052$ ).

The subcellular EGFR immunoreactivity patterns of the nine EGFR-positive corticotroph adenomas (IRS $\geq 2+$ ) are shown in Supplementary Figure 3, see section on supplementary data given at the end of this article. It is important to note that Crooke's hyaline change, characterized by the massive accumulation of cytokeratin filaments in the cytoplasm, may affect the subcellular localization of EGFR. Although one case of WT in CCA showed the cytoplasmic as well as membrane expression of EGFR, the other eight cases showed similar membrane expression patterns of EGFR, suggesting no apparent effect of USP8 mutations on the subcellular localization of EGFR.

\section{Relative mRNA expression of POMC}

In Fig. 4A, CCA showed significantly lower POMC mRNA levels than non-CCA (median 0.54 vs $1.84, P=0.012$ ). $P O M C$ mRNA expression levels were significantly higher in USP8 mutants than in WT USP8 (median 2.49 vs 0.52 , $P<0.001)$. In Fig. $4 \mathrm{~B}$, a positive correlation was observed between USP8 mRNA expression and POMC mRNA expression levels in the mutants $(r=0.60, P=0.017)$, but 
A $\quad$ WT (non-CCA+CCA) median $0.52 \quad \square P<0.001$
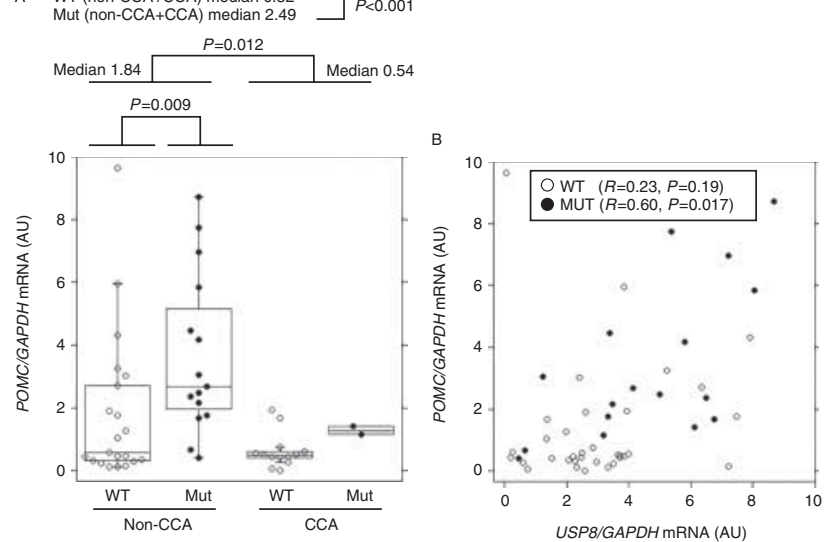

\section{Figure 4}

Expression of POMC mRNA in corticotroph adenomas.

(A) Comparison of POMC mRNA expression levels between WT and USP8 mutant corticotroph adenomas in non-CCA or CCA. Expression levels of POMC mRNA were analyzed by qRT-PCR, normalized to GAPDH, and expressed in arbitrary units (AU). (B) Relationship between relative USP8 mRNA and relative POMC mRNA levels (WT, open circles; mutant, filled circles). (C) Relationship between maximum tumor diameter and relative POMC mRNA levels. Results are presented as box plots, and the line denotes the median values. The whiskers are 5th and 95th percentiles. Significance was determined by the Mann-Whitney $U$ test (A). Spearman's rank correlation coefficient was used to calculate $R$ and $P$ values (B and $C$ ).

not in the WT $(r=0.23, P=0.19)$, indicating USP8 mutantdependent $P O M C$ mRNA expression. Maximum tumor diameters and POMC mRNA expression levels showed a negative correlation $(r=-0.54, P<0.001)$ (Fig. 4C). The stepwise regression analysis identified maximum tumor diameter and USP8 mRNA expression as contributors to $P O M C$ mRNA expression (adjusted $R^{2}=0.256, P=0.0003$ ). Taken together, small tumors such as USP8 mutants generally had higher POMC mRNA expression levels, whereas large tumors such as CCA generally had lower POMC mRNA expression levels.

\section{Relative mRNA and protein expression of somatostatin and dopamine receptors}

The mRNA and protein expression levels of SSTR2 were both generally low in the entire cohort. No significant differences were observed in SSTR2 mRNA or protein expression levels between WT and mutants or between non-CCA and CCA (Supplementary Figure 5A and B,

Mut (non-CCA+CCA) median $12+\square P<0.001$
$P=0.010$
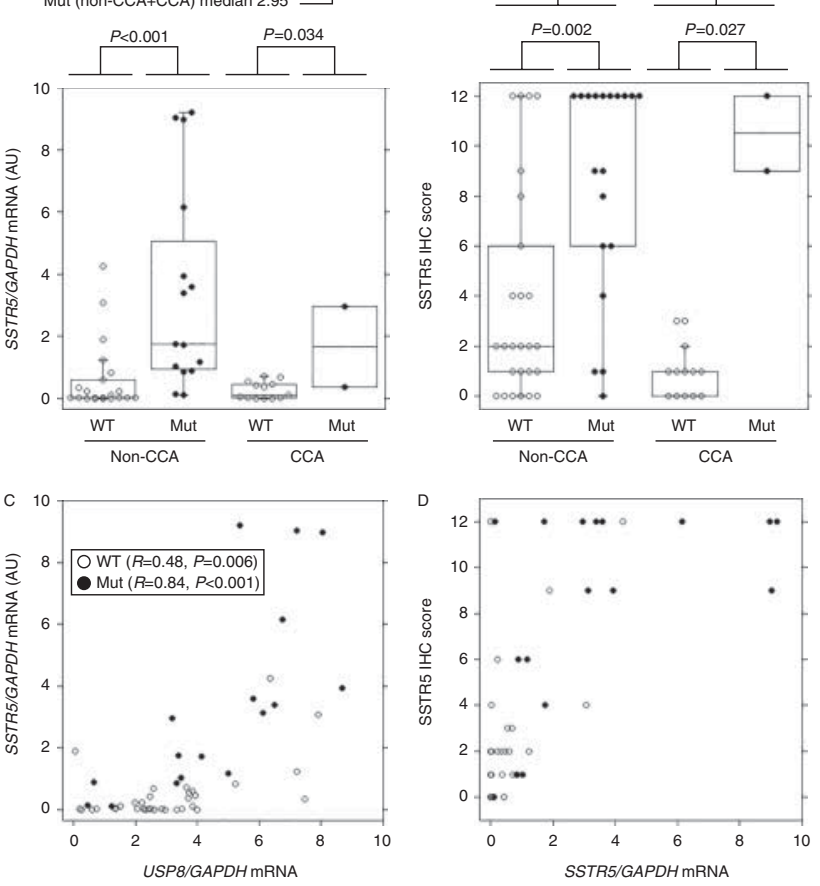

Figure 5

Expression of SSTR5 in corticotroph adenomas. (A) Comparison of SSTR5 mRNA expression levels between WT and USP8 mutant corticotroph adenomas in non-CCA or CCA. Expression levels of SSTR5 were analyzed by qRT-PCR, normalized to GAPDH, and expressed in arbitrary units (AU). (B) Comparison of SSTR5 IHC scores between WT and USP8 mutant corticotroph adenomas in non-CCA or CCA. SSTR5 staining intensity was scored using immunoreactive scores (IRS, 0-12). (C) Relationship between relative USP8 mRNA and relative SSTR5 mRNA levels (WT, open circles; mutant, filled circles). (D) Relationship between relative SSTR5 mRNA levels and SSTR5 IHC scores (WT, open circles; mutant, filled circles). Results are presented as box plots, and the line denotes the median values. The whiskers are 5th and 95th percentiles. Significance was determined by the MannWhitney $U$ test ( $A$ and $B$ ). Spearman's rank correlation coefficient was used to calculate $R$ and $P$ values (C). 
see section on supplementary data given at the end of this article).

The mRNA expression of SSTR5 was significantly higher in USP8 mutants than in WT USP8 (median 2.95 vs $0.06, P<0.001$ ) (Fig. 5A). USP8 mRNA and SSTR5 mRNA expression levels showed a positive correlation (WT: $r=0.48, P=0.006$; mutants: $r=0.84, P<0.001$ ) (Fig. 5C). The stepwise regression analysis identified the USP8 mutation status and USP8 mRNA expression as contributors to SSTR 5 mRNA expression (adjusted $R^{2}=0.561$, $P<0.0001)$. Similar to mRNA expression, SSTR5 protein expression levels was significantly higher in the mutants than in the WT (median IRS $12+$ vs $1+, P<0.001$ ) (Fig. 5B). The IHC score was significantly lower in CCA than in non-CCA (median IRS $1+$ vs $4+, P=0.010$ ) (Fig. 5B). Cases of lower IRS were mainly found in the low SSTR5 mRNA expression group of WT (Fig. 5D). The stepwise regression analysis identified the USP8 mutation status and USP8 mRNA expression as contributors to the IHC score of SSTR5 (adjusted $R^{2}=0.425, P<0.0001$ ). The subcellular SSTR5 immunoreactivity patterns of the 15 corticotroph adenomas that exhibited IRS scores of $12+$ are shown in Supplementary Figure 4, see section on supplementary data given at the end of this article. Crooke's hyaline change may have affected the subcellular localization of SSTR5. The WT and mutants showed similar membrane expression patterns of SSTR5.

In DRD2, mRNA and protein expression levels were both generally low in the entire cohort. However, DRD2 mRNA expression levels were significantly higher in the USP8 mutants of CCA than in the WT of CCA (median 0.81 vs $0.08, P=0.038$ ) (Supplementary Figure $5 \mathrm{C}$, see section on supplementary data given at the end of this article). No significant difference was observed in protein expression levels between the WT and mutants or between non-CCA and CCA (Supplementary Figure 5D).

\section{Relative mRNA and protein expression of MGMT}

MGMT mRNA expression levels were significantly higher in USP8 mutants than in the WT (median 3.47 vs 0.78 , $P=0.001$ ) (Fig. 6A). In the IHC analysis, MGMT protein expression levels were significantly higher in the mutants than in the WT (median $8+$ vs $1+, P<0.0001$ ), and most USP8 mutants (20 out of 21) were immunopositive for MGMT (IHC score $\geq 2+$ ) (Fig. 6B). MGMT mRNA and protein expression levels were significantly lower in CCA than in non-CCA, and most CCA (13 out of 15) were immunonegative for MGMT (Fig. 6A and B). USP8 mRNA expression and MGMT mRNA expression levels showed

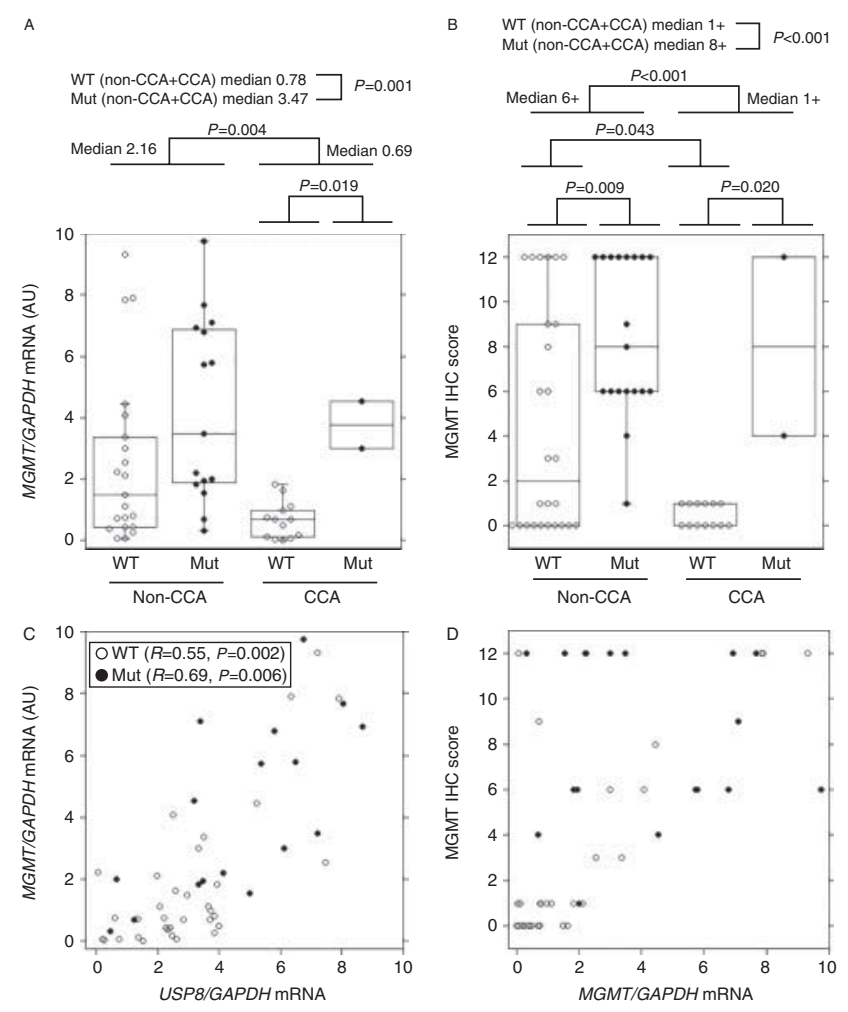

\section{Figure 6}

Expression of MGMT in corticotroph adenomas. (A) Comparison of MGMT mRNA expression levels between WT and USP8 mutant corticotroph adenomas in non-CCA or CCA. Expression levels of MGMT were analyzed by qRT-PCR, normalized to GAPDH, and expressed in arbitrary units (AU). (B) Comparison of MGMT IHC scores between WT and USP8 mutant corticotroph adenomas in non-CCA or CCA. MGMT staining intensity was scored using immunoreactive scores (IRS, 0-12). (C) Relationship between relative USP8 mRNA and relative MGMT mRNA levels (WT, open circles; mutant, filled circles). (D) Relationship between relative MGMT mRNA levels and MGMT IHC scores (WT, open circles; mutant, filled circles). Results are presented as box plots, and the line denotes the median values. The whiskers are 5th and 95th percentiles. Significance was determined by the Mann-Whitney $U$ test ( $A$ and $B$ ). Spearman's rank correlation coefficient was used to calculate $R$ and $P$ values (C).

a positive correlation (WT: $r=0.55, P=0.002$; mutants: $r=0.69, P=0.006$ ) (Fig. 6C). MGMT immunonegative tumors were mainly detected in the lower MGMT mRNA expression group of the WT (Fig. 6D). The stepwise regression analysis identified USP8 mRNA expression levels and pathological findings (non-CCA or CCA) as contributors to MGMT mRNA expression (adjusted $R^{2}=0.579, P<0.0001$ ), and USP8 mRNA expression levels, 
the maximum tumor diameter, and pathological findings (non-CCA or CCA) as contributors to the IHC score of MGMT (adjusted $R^{2}=0.454, P<0.0001$ ).

\section{Relative mRNA and protein expression of MSH6}

MSH6 mRNA expression levels were significantly higher in USP8 mutants and non-CCA than in the WT and CCA respectively (Supplementary Figure 5E, see section on supplementary data given at the end of this article). However, in the IHC analysis, most cases in the entire cohort (57 out of 60) showed high protein expression levels $(12+)$, and there was no significant difference in protein expression levels between the WT and mutants or between non-CCA and CCA (Supplementary Figure 5F).

\section{Discussion}

We herein detected USP8 mutations in 21 out of 60 (35\%) Japanese patients with CD. Similar to previously reported mutants, new mutants (S719P, S718F, and S718_P720delinsT) lost 14-3-3 protein binding, underwent cleavage and catalytic activation, and suppressed EGFR downregulation. Among the three mutants examined, S718F was more prone to cleavage and exhibited higher DUB activity than the others, which is consistent with the critical role of Ser718 phosphorylation in USP8 binding to 14-3-3 proteins (26) and high frequency of mutations at Ser718 in corticotroph adenomas $(9,10,11)$.

The phenotype of typical USP8 mutants represents a CD with small adenomas in middle-aged women. It may have caused the higher surgical remission rate of the mutants (95.2\%) than that of the WT (53.8\%) in our subjects. Similar to our results, a Chinese group showed that mutated adenomas were significantly smaller than WT adenomas (10), whereas a German group reported no significant differences (11). The reason(s) for the discrepancy are unclear but may be partly due to differences in sample recruitment. Specifically, samples were collected from one institution in our study and the Chinese study, but from seven institutions in the German study. The ethnic difference of the subjects may also have been a factor.

Using the three new USP8 mutants, we confirmed in vitro that these mutations elevated DUB activity, thereby increasing the cell surface expression of EGFR ( 9 , 10). However, there was no significant difference in EGFR protein expression levels between the WT and mutants in our IHC analysis. The monoclonal EGFR antibody, clone 31G7 used in our study is a validated antibody widely used in IHC analyses of various cancers $(27,28)$. In our hospital, we routinely use this antibody for IHC of cancers such as colorectal cancer and breast cancer, suggesting that false negative errors were unlikely. The in vitro and in vivo discrepancies may have partly been due to the use of a supra-physiological concentration $(100 \mathrm{ng} / \mathrm{ml})$ of EGF in the in vitro experiments, which activated all EGFR molecules simultaneously. As the USP8 mutants selectively inhibit the downregulation of ligand-activated, ubiquitinated EGFR, but do not affect inactive EGFR, the total EGFR protein level may not reflect the difference in the downregulation rate of activated EGFR in vivo. Therefore, further studies are warranted. In contrast, EGFR protein expression levels were significantly higher in CCA than in non-CCA in our IHC analysis. Thus, the aggressive phenotype of CCA may be partly explained by EGFRmediated activation. In this regard, molecular targeted therapies such as TK inhibitors may be an alternate therapeutic option for refractory cases of CCA (17).

USP8 mRNA expression levels were higher in USP8mutant than in USP8-WT corticotroph adenomas (Supplementary Figure 1C, see section on supplementary data given at the end of this article). Consistent with this result, USP8 protein expression levels were shown to be higher in USP8-mutant than in WT corticotroph adenomas (9). A previous study reported that a dexamethasone treatment increased USP8 protein expression levels in a glucocorticoid receptor-dependent manner in human embryonic kidney cells, suggesting the regulation of corticotroph USP8 expression by the positive feedback system of the hypothalamic-pituitary-adrenal gland axis (29). However, this does not explain why USP8-mutant adenomas expressed USP8 mRNA more strongly than WT adenomas because no significant differences were observed in serum cortisol levels between mutant and WT adenomas (Table 1). Therefore, the molecular mechanisms underlying the upregulation of USP8 gene expression by its mutations in the protein-coding region (i.e. 14-3-3 binding motif) remain unknown.

We showed that mRNA and protein expression levels of SSTR5 were significantly higher in the USP8 mutant group than in the WT group. Information regarding the mechanisms of transcriptional regulation for the SSTR5 gene is currently limited. It will be interesting to determine in vitro whether USP8 mutants directly regulate SSTR5 mRNA expression. However, the elevated expression of SSTR5 mRNA in USP8-mutant adenomas may be an epiphenomenon to the tumor phenotype of the mutants. The higher SSTR5 mRNA levels of the mutants may not solely explain the higher SSTR5 protein 
expression levels. SSTRs belong to the superfamily of G protein-coupled receptors (GPCRs). Similar to other GPCRs, agonist-stimulated SSTRs undergo desensitization and endocytosis (30). Recent studies revealed that the desensitization of GPCRs is mediated not only by phosphorylation and $\beta$-arrestins, but also by ubiquitination (31). Of particular interest is whether ubiquitinated SSTR5 is a substrate of USP8 because the elevated DUB activity of the USP8 mutants may inhibit the downregulation of SSTR5.

The somatostatin analog pasireotide, which possesses high SSTR5 affinity, has been reported to normalize urinary cortisol levels in $\sim 25-30 \%$ of patients with CD $(12,32,33,34)$. The higher SSTR5 expression levels in USP8 mutant adenomas suggest that the presence of USP8 mutations may predict favorable responses to pasireotide in addition to their high surgical remission rate shown in the present study. In contrast, surgically refractory cases of USP8 WT adenomas including CCA, which often present with invasive macroadenomas, may generally be resistant to medical therapy with pasireotide.

MGMT mRNA and protein expression levels were both significantly higher in USP8 mutants than in the WT. Furthermore, compared with the WT, strong immunopositive expression of MGMT was generally observed in the mutants, even when their MGMT mRNA expression levels were low. The MGMT protein was shown to be degraded via the ubiquitin-proteasome pathway $(35,36)$. Therefore, USP8 may play a role in the deubiquitination of MGMT. In this scenario, MGMT degradation is inhibited when adenomas either abundantly express USP8 or have USP8 mutations, leading to positive MGMT protein expression. However, elevated MGMT mRNA expression may be due to a USP8 mutation-triggered epiphenomenon.

The alkylating compound, temozolomide, recently demonstrated a significant response against aggressive pituitary adenomas and carcinomas $(19,37,38)$. A recent relatively large cohort study $(n=24)$ (19) demonstrated that immunohistochemically low MGMT expression predicts a better response to temozolomide. In our series of $\mathrm{CD}$, most CCA exhibited immunohistochemically negative MGMT expression, which was consistent with our previous findings (21). Although aggressive tumors including CCA are not good therapeutic candidates for pasireotide, such patients may have a better response to temozolomide.

Several studies have argued that an immunohistochemical MGMT expression analysis is not clinically useful in predicting tumor responses to temozolomide therapy $(20,37,38)$. One possible explanation of the controversy is the acquisition of temozolomide resistance due to the loss of MSH6 expression during temozolomide treatments $(19,20,39)$. In our subjects, most patients (58 out of 60) were immunopositive for MSH6. Therefore, care is needed regarding the loss of MSH6 in acquiring temozolomide resistance after starting temozolomide treatments.

In summary, USP8 mutations were frequently detected in females and smaller tumors. Microadenomas that strongly express $P O M C$ were common among the mutated tumors, which may provide an insight into the mechanisms by which microadenomas secrete excess ACTH in order to present overt CD. The frequency of USP8 mutations was also significantly greater in tumors expressing SSTR5, suggesting favorable responses to the somatostatin analog pasireotide. In contrast, non-mutated aggressive tumors such as CCA may respond better to the alkylating agent temozolomide because of their significantly weak expression of MGMT. Although EGFR is theoretically activated in USP8 mutant adenomas, we failed to demonstrate this in vivo. Further studies will be necessary to clarify this issue.

\section{Supplementary data}

This is linked to the online version of the paper at http://dx.doi.org/10.1530/ EJE-15-0689.

\section{Declaration of interest}

The authors declare that there is no conflict of interest that could be perceived as prejudicing the impartiality of the research reported.

\section{Funding}

This work was supported by the Okinaka Memorial Institute for Medical Research to A Takeshita. JSPS KAKENHI (grant number 25281024 to A Takeshita, 25460468 to N Inoshita, and 24112003 to M Komada).

\section{References}

1 George DH, Scheithauer BW, Kovacs K, Horvath E, Young WF Jr, Lloyd RV \& Meyer FB. Crooke's cell adenoma of the pituitary: an aggressive variant of corticotroph adenoma. American Journal of Surgical Pathology 200327 1330-1336. (doi:10.1097/00000478200310000-00005)

2 Di Ieva A, Davidson JM, Syro LV, Rotondo F, Montoya JF, Horvath E, Cusimano MD \& Kovacs K. Crooke's cell tumors of the pituitary. Neurosurgery 201576 616-622. (doi:10.1227/NEU.0000000000000657)

3 Yarden Y \& Pines G. The ERBB network: at last, cancer therapy meets systems biology. Nature Reviews. Cancer 201212 553-563. (doi:10.1038/ nrc3309) 
4 Kontogeorgos G, Stefaneanu L, Kovacs K \& Cheng Z. Localization of epidermal growth factor (EGF) and epidermal growth factor receptor (EGFr) in human pituitary adenomas and nontumorous pituitaries: an immunocytochemical study. Endocrine Pathology 19967 63-70. (doi:10.1007/BF02739916)

5 Theodoropoulou M, Arzberger T, Gruebler Y, Jaffrain-Rea ML, Schlegel J, Schaaf L, Petrangeli E, Losa M, Stalla GK \& Pagotto U. Expression of epidermal growth factor receptor in neoplastic pituitary cells: evidence for a role in corticotropinoma cells. Journal of Endocrinology 2004183 385-394. (doi:10.1677/joe.1.05616)

6 LeRiche VK, Asa SL \& Ezzat S. Epidermal growth factor and its receptor (EGF-R) in human pituitary adenomas: EGF-R correlates with tumor aggressiveness. Journal of Clinical Endocrinology and Metabolism $1996 \mathbf{8 1}$ 656-662. (doi:10.1210/jcem.81.2.8636285)

7 Onguru O, Scheithauer BW, Kovacs K, Vidal S, Jin L, Zhang S, Ruebel KH $\&$ Lloyd RV. Analysis of epidermal growth factor receptor and activated epidermal growth factor receptor expression in pituitary adenomas and carcinomas. Modern Pathology 200417 772-780. (doi:10.1038/ modpathol.3800118)

8 Cooper O, Vlotides G, Fukuoka H, Greene MI \& Melmed S. Expression and function of ErbB receptors and ligands in the pituitary. EndocrineRelated Cancer 201118 R197-R211. (doi:10.1530/ERC-11-0066)

9 Reincke M, Sbiera S, Hayakawa A, Theodoropoulou M, Osswald A, Beuschlein F, Meitinger T, Mizuno-Yamasaki E, Kawaguchi K, Saeki Y et al. Mutations in the deubiquitinase gene USP8 cause Cushing's disease. Nature Genetics 201547 31-38. (doi:10.1038/ng.3166)

10 Ma ZY, Song ZJ, Chen JH, Wang YF, Li SQ, Zhou LF, Mao Y, Li YM, Hu RG, Zhang ZY et al. Recurrent gain-of-function USP8 mutations in Cushing's disease. Cell Research 201525 306-317. (doi:10.1038/cr. 2015.20)

11 Perez-Rivas LG, Theodoropoulou M, Ferrau F, Nusser C, Kawaguchi K, Stratakis CA, Rueda Faucz F, Wildemberg LE, Assie G, Beschorner R et al. The gene of the ubiquitin-specific protease 8 is frequently mutated in adenomas causing Cushing's disease. Journal of Clinical Endocrinology and Metabolism 2015100 E997-E1004. (doi:120.120/jc.2015-1453)

12 Feelders RA \& Hofland LJ. Medical treatment of Cushing's disease. Journal of Clinical Endocrinology and Metabolism 201398 425-438. (doi:10.1210/jc.2012-3126)

13 Gadelha MR \& Vieira Neto L. Efficacy of medical treatment in Cushing's disease: a systematic review. Clinical Endocrinology 201480 1-12. (doi:10.1111/cen.12345)

14 Ortiz LD, Syro LV, Scheithauer BW, Rotondo F, Uribe H, Fadul CE, Horvath E \& Kovacs K. Temozolomide in aggressive pituitary adenomas and carcinomas. Clinics 201267 (Suppl 1) 119-123. (doi:10.6061/ clinics/2012(Sup01)20)

15 Di Ieva A, Rotondo F, Syro LV, Cusimano MD \& Kovacs K. Aggressive pituitary adenomas-diagnosis and emerging treatments. Nature Reviews. Endocrinology 201410 423-435. (doi:10.1038/nrendo.2014.64)

16 Chatzellis E, Alexandraki KI, Androulakis II \& Kaltsas G. Aggressive pituitary tumors. Neuroendocrinology 2015101 87-104. (doi:10.1159/ 000371806)

17 Fukuoka H, Cooper O, Ben-Shlomo A, Mamelak A, Ren SG, Bruyette D $\&$ Melmed S. EGFR as a therapeutic target for human, canine, and mouse ACTH-secreting pituitary adenomas. Journal of Clinical Investigation 2011121 4712-4721. (doi:10.1172/JCI60417)

18 McCormack AI, Wass JA \& Grossman AB. Aggressive pituitary tumours: the role of temozolomide and the assessment of MGMT status. European Journal of Clinical Investigation 201141 1133-1148. (doi:10.1111/ j.1365-2362.2011.02520.x)

19 Bengtsson D, Schroder HD, Andersen M, Maiter D, Berinder K, Feldt Rasmussen U, Rasmussen AK, Johannsson G, Hoybye C \& van der Lely AJ. Long-term outcome and MGMT as a predictive marker in 24 patients with atypical pituitary adenomas and pituitary carcinomas given treatment with temozolomide. Journal of Clinical Endocrinology and Metabolism 2015100 1689-1698. (doi:10.1210/jc.2014-4350)
20 Hirohata T, Asano K, Ogawa Y, Takano S, Amano K, Isozaki O, Iwai Y, Sakata K, Fukuhara N, Nishioka $\mathrm{H}$ et al. DNA mismatch repair protein (MSH6) correlated with the responses of atypical pituitary adenomas and pituitary carcinomas to temozolomide: the national cooperative study by the Japan Society for Hypothalamic and Pituitary Tumors. Journal of Clinical Endocrinology and Metabolism 201398 1130-1136. (doi:10.1210/jc.2012-2924)

21 Takeshita A, Inoshita N, Taguchi M, Okuda C, Fukuhara N, Oyama K, Ohashi K, Sano T, Takeuchi Y \& Yamada S. High incidence of low $\mathrm{O}^{6}$-methylguanine DNA methyltransferase expression in invasive macroadenomas of Cushing's disease. European Journal of Endocrinology 2009161 553-559. (doi:10.1530/EJE-09-0414)

22 Yamada S, Fukuhara N, Nishioka H, Takeshita A, Inoshita N, Ito J \& Takeuchi Y. Surgical management and outcomes in patients with Cushing disease with negative pituitary magnetic resonance imaging. World Neurosurgery 201277 525-532. (doi:10.1016/j.wneu. 2011.06.033)

23 Findling JW \& Raff H. Cushing's syndrome: important issues in diagnosis and management. Journal of Clinical Endocrinology and Metabolism 200691 3746-3753. (doi:10.1210/jc.2006-0997)

24 Lupp A, Hunder A, Petrich A, Nagel F, Doll C \& Schulz S. Reassessment of sst(5) somatostatin receptor expression in normal and neoplastic human tissues using the novel rabbit monoclonal antibody UMB-4. Neuroendocrinology 201194 255-264. (doi:10.1159/000329876)

25 Casar-Borota O, Heck A, Schulz S, Nesland JM, Ramm-Pettersen J, Lekva T, Alafuzoff I \& Bollerslev J. Expression of SSTR2a, but not of SSTRs 1, 3, or 5 in somatotroph adenomas assessed by monoclonal antibodies was reduced by octreotide and correlated with the acute and long-term effects of octreotide. Journal of Clinical Endocrinology and Metabolism 201398 E1730-E1739. (doi:10.1210/jc.2013-2145)

26 Mizuno E, Kitamura N \& Komada M. 14-3-3-dependent inhibition of the deubiquitinating activity of UBPY and its cancellation in the M phase. Experimental Cell Research 2007313 3624-3634. (doi:10.1016/j.yexcr.2007.07.028)

27 Reis-Filho JS, Milanezi F, Carvalho S, Simpson PT, Steele D, Savage K, Lambros MB, Pereira EM, Nesland JM, Lakhani SR et al. Metaplastic breast carcinomas exhibit EGFR, but not HER2, gene amplification and overexpression: immunohistochemical and chromogenic in situ hybridization analysis. Breast Cancer Research 20057 R1028-R1035. (doi:10.1186/bcr1341)

28 Bhargava R, Chen B, Klimstra DS, Saltz LB, Hedvat C, Tang LH, Gerald W, Teruya-Feldstein J, Paty PB, Qin J et al. Comparison of two antibodies for immunohistochemical evaluation of epidermal growth factor receptor expression in colorectal carcinomas, adenomas, and normal mucosa. Cancer 2006106 1857-1862. (doi:10.1002/ cncr.21782)

29 Sippel M, Rajala R, Korhonen L, Bornhauser B, Sokka AL, Naito M \& Lindholm D. Dexamethasone regulates expression of BRUCE/Apollon and the proliferation of neural progenitor cells. FEBS Letter 2009583 2213-2217. (doi:10.1016/j.febslet.2009.06.018)

30 Lesche S, Lehmann D, Nagel F, Schmid HA \& Schulz S. Differential effects of octreotide and pasireotide on somatostatin receptor internalization and trafficking in vitro. Journal of Clinical Endocrinology and Metabolism 200994 654-661. (doi:10.1210/jc.2008-1919)

31 Alonso V \& Friedman PA. Minireview: ubiquitination-regulated G protein-coupled receptor signaling and trafficking. Molecular Endocrinology 201327 558-572. (doi:10.1210/me.2012-1404)

32 Boscaro M, Ludlam WH, Atkinson B, Glusman JE, Petersenn S, Reincke M, Snyder P, Tabarin A, Biller BM, Findling J et al. Treatment of pituitary-dependent Cushing's disease with the multireceptor ligand somatostatin analog pasireotide (SOM230): a multicenter, phase II trial. Journal of Clinical Endocrinology and Metabolism 200994 115-122. (doi:10.1210/jc.2008-1008)

33 Colao A, Petersenn S, Newell-Price J, Findling JW, Gu F, Maldonado M, Schoenherr U, Mills D, Salgado LR \& Biller BM. A 12-month phase 3 
study of pasireotide in Cushing's disease. New England Journal of Medicine 2012366 914-924. (doi:10.1056/NEJMoa1105743)

34 Ceccato F, Scaroni C \& Boscaro M. Clinical use of pasireotide for Cushing's disease in adults. Therapeutics and Clinical Risk Management 201511 425-434. (doi:10.2147/tcrm.s37314)

35 Srivenugopal KS, Yuan XH, Friedman HS \& Ali-Osman F. Ubiquitination-dependent proteolysis of $\mathrm{O}^{6}$-methylguanine-DNA methyltransferase in human and murine tumor cells following inactivation with $\mathrm{O}^{6}$-benzylguanine or 1,3-bis(2-chloroethyl)-1nitrosourea. Biochemistry 199635 1328-1334. (doi:10.1021/bi9518205)

36 Xu-Welliver M \& Pegg AE. Degradation of the alkylated form of the DNA repair protein, $\mathrm{O}^{6}$-alkylguanine-DNA alkyltransferase. Carcinogenesis 200223 823-830. (doi:10.1093/carcin/23.5.823)

37 Raverot G, Sturm N, de Fraipont F, Muller M, Salenave S, Caron P, Chabre O, Chanson P, Cortet-Rudelli C, Assaker R et al. Temozolomide treatment in aggressive pituitary tumors and pituitary carcinomas: a French multicenter experience. Journal of Clinical Endocrinology and Metabolism 201095 4592-4599. (doi:10.1210/jc.2010-0644)

38 Bush ZM, Longtine JA, Cunningham T, Schiff D, Jane JA Jr, Vance ML, Thorner MO, Laws ER Jr \& Lopes MB. Temozolomide treatment for aggressive pituitary tumors: correlation of clinical outcome with $\mathrm{O}^{6}$-methylguanine methyltransferase (MGMT) promoter methylation and expression. Journal of Clinical Endocrinology and Metabolism 201095 E280-E290. (doi:10.1210/jc.2010-0441)

39 Murakami M, Mizutani A, Asano S, Katakami H, Ozawa Y, Yamazaki K, Ishida $\mathrm{Y}$, Takano $\mathrm{K}$, Okinaga $\mathrm{H} \&$ Matsuno A. A mechanism of acquiring temozolomide resistance during transformation of atypical prolactinoma into prolactin-producing pituitary carcinoma: case report. Neurosurgery 201168 E1767; discussion E1767. (doi:10.1227/NEU. 0b013e318217161a)

Received 9 July 2015

Revised version received 15 October 2015

Accepted 16 November 2015 